\title{
Changes in activities of daily living (ADL) among elderly Chinese by marital status, living arrangement, and availability of healthcare over a 3-year period
}

\author{
Dewen Wang · Jianmin Zheng • Michiko Kurosawa • \\ Yutaka Inaba · Noriko Kato
}

Received: 24 April 2008/Accepted: 9 December 2008/Published online: 20 January 2009

(c) The Japanese Society for Hygiene 2009

\begin{abstract}
Objectives The purpose of this study was to assess how changes from different baselines of activities of daily living (ADL) can be explained by marital status, living arrangement and healthcare.

Methods Using data from the Chinese Longitudinal Health Longevity Study conducted in 2002 and 2005, 8,099 surviving and 3,822 deceased elderly aged 65 years and over were evaluated using multinomial logistic regression. Results After adjusting for demographic, socioeconomic and health factors, elderly who were either married or living alone were less likely to encounter ADL decline compared to their counterparts. This was true only for those with fully independent ADL at baseline. Notably, once the functional status of the elderly declined from baseline and they became dependent on others, the status of living alone was no longer a significant predictor of the rate of future decline. On the other hand, elderly who had a spouse, children or other relatives as caregivers were more likely to
\end{abstract}

\section{Wang $(\bowtie)$}

Institute of Population Research, Xiamen University, 361005 Xiamen, China

e-mail: dewen88@xmu.edu.cn; tou@juntendo.ac.jp

D. Wang · M. Kurosawa · Y. Inaba

Department of Epidemiology and Environmental Health,

School of Medicine, Juntendo University,

Tokyo 113-8421, Japan

D. Wang · N. Kato

Department of Social Welfare,

National Institute of Public Health, Saitama, Japan

J. Zheng

Department of Psychology,

First Affiliated Hospital of Fujian Medical University,

Fuzhou, China experience a faster recovery and lower likelihood of death, compared to those who were cared by unrelated live-in caregivers. In addition, Chinese elderly with health insurance had a lower likelihood of death than their counterparts lacking health insurance, among those with ADL at the dependent baseline.

Conclusions Although there has been a change in family structure and living arrangements, the majority of Chinese elderly still rely on traditional forms of family support, especially after acquiring dependency status. As the elderly have different functional levels, healthcare policies in China should consider the need for both community and family support systems.

Keywords Elderly in China - Change in ADL . Marital status $\cdot$ Living arrangement $\cdot$ Health care

\section{Introduction}

In China, a rapid increase is predicted for the near future in the proportion of elderly people in the population, due to the one-child policy. Populations in many countries including China are aging, and change in family structure and living arrangements of the elderly is apparent. Some studies [1, 2] based on sampling data from censuses in China indicated that Chinese families are turning from a traditional to a modern structure. For example, an increase in people living alone, empty-nest households, and nuclear family households has been observed, while multi-generational family households have decreased. In addition, growing independence of married couples from their older relatives, democratization of the family relationships, and socialization of family functions have occurred, due to economic development, urbanization, and other sociological factors 
$[2,3]$. These recent changes in critical demographic factors in China have therefore generated concern for improving the health status and living circumstances of the elderly.

Studies conducted in western countries have shown that household structure can affect the incidence of decline in functional status of the elderly. For example, a study conducted among urban, non-institutionalized individuals at least 65 years of age showed that elderly who lived with children and without spouses had worse health outcomes, compared to elderly living alone [4]. Another study showed that people who lived with a partner had lower morbidity rates than did people who lived alone; after controlling for the effects of different living arrangements, the risks for all objective measures of illness of the 'never married', widowed and divorced were $40-70 \%$ less than the risks for people who lived with a partner [5]. A more recent, prospective study of women with no severe impairment at a baseline level suggested that those living alone were less likely to experience a decline in functional status than were those living with spouses or other people [6].

Although a few studies have focused on changes in family support networks for the elderly in China [7], little is known regarding how the marital status, living arrangements and healthcare issues may affect changes in their functional status. Understanding the influence of these factors is likely to be useful in promoting family support networks and improving health of the elderly. The purpose of the current study is to determine whether changes in activities of daily living (ADL) of Chinese elderly possessing different baseline ADL levels can be explained by marital status, living arrangements and nature of caregivers.

\section{Methods}

Database

The Chinese Longitudinal Health Longevity Study (CLHLS) [8, 9] was conducted in 1998 (baseline), and follow-up studies were performed in 2000, 2002, and 2005. The current study used the third and fourth waves (2002 and 2005, respectively) of the CLHLS. The CLHLS was conducted in randomly selected $50 \%$ of the counties and cities in 22 provinces. The 22 surveyed provinces were: Liaoning, Jilin, Heilongjiang, Hebei, Beijing, Tianjing, Shanxi, Shaanxi, Shanghai, Jiangsu, Zhejiang, Anhui, Fujian, Jiangxi, Shandong, Henan, Hubei, Hunan, Guangdong, Guangxi, Sichuan, and Chongqing, which have a total population of 985 million and represent $85 \%$ of the total population in China.

The CLHLS survey tried to interview all centenarians from the above counties and cities who voluntarily agreed to participate in the study. Starting with the third wave of the CLHLS in 2002, for each centenarian, the surveyed elderly included subjects in an age range of $65-79$, one octogenarian (i.e., 80-89 years of age) and one nonagenarian (i.e., 90-99 years of age) of a pre-designated age and gender who lived nearby. "Nearby" was loosely defined as the same street, village, town, city, or county. The predefined age and gender used to identify approximately equal numbers of male and female in each age group were randomly determined, based on the code numbers of the centenarians. One goal of the CLHLS was to have comparable numbers of male and female of each age from 65 to 99 .

The CLHLS in 2002 comprised a total sample size of 16,057 subjects, of which 4,894 elderly were aged 65-79, 4,210 were aged 80-89, 3,632 were aged 90-99, and 3,321 were aged 100 years and above. The follow-up survey conducted in 2005 contained data for 8,136 subjects who were interviewed in the follow-up, and for 4,111 deceased subjects whose families were interviewed. The population eligible for our study was defined as subject's $\geq 65$ years of age who were interviewed during the follow-up periods in 2002 and 2005. Our total study sample was 8,099 surviving interviewees and 3,822 deceased interviewees.

An extensive questionnaire (containing 92 questions pertaining to 180 items) was used in the CLHLS. Data were collected on demographic factors, socioeconomic factors, living arrangements, and health indicators, such as the ADL and the mini-mental state examination (MMSE) etc. For the CLHLS, an enumerator and either a doctor, nurse, or medical student conducted an interview and performed a basic health examination at the interviewee's home $[9,10]$.

\section{Measures}

The six basic activities in the ADL consist of eating, dressing, indoor mobility, bathing, using the toilet, and continence. While considering the ADL measurement method proposed by Katz [11], the following measurement criteria were developed for the present study. The degrees of function in ADL were categorized as: fully independent (FI; i.e., no assistance required); relatively independent (RI; i.e., requiring assistance for only one or two activities); relatively dependent (RD; i.e., requiring assistance in three or four activities); and fully dependent (FD; i.e., requiring assistance in five or six activities). In addition, the change in ADL was also analyzed among the deceased interviewees as one of the severe forms of decline in physical functions.

Exposure variables were measured at baseline (i.e., in 2002), except for the frequency of serious illness in the past 2 years among the surviving interviewees (e.g., in 2005). Marital status was transformed into a dichotomous 
variable, indicating married versus single (i.e., widowed, divorced, separated or never married). Living arrangements were identified through the marital status of the individual, the household size, and the co-residence members. Thus, the potential types of living arrangements were "living in a nursing home", "living alone", "living with spouse only" (specified by a household size of two, a married status, and the co-resident member being the spouse), and "living with others" (defined by a household size $\geq 3$ individuals). For the variable of living arrangements, the term, "others" was defined as a spouse, children, grandchildren, or other relatives. In our study, the category of an elderly person living with a child or children younger than 18 years of age was deleted, because the limited number of subjects that fell into that category made the particular living arrangement difficult to evaluate.

Our study included the following healthcare questions: (1) who (spouse, children, relatives, live-in caregivers, and/ or others) usually takes care of you when you are ill? (2) Can you obtain adequate medical service when you are ill? (yes or no). (3) "Who pays your medical bills for you?" (i.e., having health insurance, family, relatives or others). This question was asked to identify whether a subject had health insurance or not.

For accuracy in investigating the role of the factors of marital status, living arrangements, and healthcare issues on changes in categories of ADL, we eliminated confounding factors including demographic, socioeconomic and health factors. Health factors included cognitive ability and frequency of serious illness within the past 2 years. Demographic and socioeconomic factors included gender, age, residence, education, occupation, and household income. The number of years of schooling was used to represent the educational level of the elderly subjects. We pre-processed the data on years of schooling into the following categories, according to their distribution: "no education" (i.e., illiteracy), 1-4 years of schooling, and $\geq 5$ years of schooling. We divided occupational data into agricultural or non-agricultural, according to the subjects' occupations before they were 60 years of age. This division was made because nearly two-thirds of the current population of elderly people in China were involved in farming and agricultural production. Household income was preprocessed into the following three categories according to distribution: $<1,200$ RMB per capita per year, 1,201-3,000 RMB per capita per year, and $>3,001$ RMB per capita per year.

The international standard of the MMSE questionnaire used in this study to measure cognitive ability was translated into Chinese [9]. The same parameters used in the MMSE international standard were applied here; thus, a score of 0-30 was possible (i.e., the lower the score, the more severe was the cognitive impairment). The frequency of serious illness requiring hospitalization or that which caused the subject to be bedridden at home within the past 2 years was calculated for the year 2005 . We pre-processed an extended period of time that for which a subject was bedridden at home as a score of 24 , representing 24 months ( 2 years). The distribution of frequency of serious illness requiring hospitalization ranged from 0 to 16 . Thus, frequency of serious illness and period of being bedridden at home were quantitative variables in the analyses.

\section{Statistical analyses}

Statistical analyses including a $\chi^{2}$ test or Kruskal-Wallis $\mathrm{H}$ test were conducted to analyze the change in ADL and either the exposure variables (marital status, living arrangements, and availability of healthcare) or the main confounding variables (i.e., demographic, socioeconomic and health factors). Multinomial logistic regression analysis was run using different categories of ADL (including dead) as a dependent variable. Three multinomial logistic regression models were used to test the influence of marital status, living arrangements, and availability of healthcare on changes in ADL from the different baselines for elderly subjects in China. The first model estimated the relative risk factors of progressing from FI to RI, RD, FD, and dying among those at FI baseline. The second and third models incorporated the relative factors of progressing those from RI or RD and FD to FI or FI and RI, RD and $\mathrm{FD}$, and dying among those at RI or RD and FD baseline, respectively. Due to a limited number of cases in some categories, those who were RD and FD were combined in the analysis; for the same reason, FI and RI were also combined when the onset of ADL at RD and FD baselines. Analyses were performed with SPSS Version 16.0 statistical software.

\section{Results}

Our total study sample was 11,921 elderly people, Of these, $5,487(46 \%)$ were male, and 6,434 (54\%) were female; $6,491(54 \%)$ of subjects resided in the rural countryside of China, and 5,430 (46\%) resided in towns and cities in China; 7,137 (60\%) elderly subjects with no education, 2,451 (21\%) with 1-4 years schooling, 2,275 (19\%) with $5+$ years schooling (with 58 case missing); 3,812 (32\%) elderly subjects with household income less than 1,200 yuan per year per person, 3,656 (31\%) with $1,201-3,000$ yuan per year per person, $3,900(33 \%)$ with $3,000+$ yuan per year per person (with 553 cases missing); 4,703 (39\%) elderly person not in agriculture before 60 years, 7,169 (60\%) in agriculture (with 49 cases missing); 4,174 (35\%) elderly subjects were currently married, and 7,746 (65\%) 
were single (with 1 case missing). The average age of male subjects was $82.31 \pm 10.50$ years, and that of females' was $86.40 \pm 11.79$ years. On the other hand, the average age of the currently married elderly was $76.79 \pm 8.90$ years, and that for the single elderly was $88.81 \pm 10.36$ years. In addition, among the 4,174 elderly who were currently married and living with a spouse, 2,838 (68\%) were men whose average age was $77.92 \pm 9.21$ years, and 1,336 $(32 \%)$ were women whose average age was $74.51 \pm$ 7.79 years. Among 2,121 elderly who lived alone, 805 (38\%) were men whose average age was $84.52 \pm 9.96$ years, whereas $1,316(62 \%)$ were women whose average age was $85.22 \pm 10.95$ years.

Characteristics of the study population according to the changes in ADL among the elderly from 2002 to 2005 are presented in Table 1. Regarding changes in ADL from 2002 to $2005: 8,857$ (75\%) elderly subjects were FI, 1,834 (15\%) were RI, 398 (3\%) were RD, and 832 (7\%) were FD in 2002. Among 8,857 subjects who were FI at baseline, 5,694 (64.3\%) maintained FI, $581(6.6 \%)$ declined to RI, $153(1.7 \%)$ declined to RD, 333 (3.8\%) declined to FD, and 2,096 (23.7\%) had died; while among 1,834 subjects who were RI at baseline, 402 (21.9\%) improved from RI to FI, $315(17.2 \%)$ maintained RI, 250 (13.6\%) declined to RD or FD, and 867 (47.3\%) had died; and among 1,230 subjects who were RD and FD at baseline, 179 (14.6\%) improved from RD or FD to RI or FI, 192 (15.6\%) maintained RD or FD, and 859 (69.8\%) had died.

Among subjects who were FI at baseline, significant correlations with changes in ADL were found with all categories of demographic variables, socioeconomic factors, the exposure variables (with the exception of having health insurance) and factors of health status. However, for those who were RI at baseline, there were no statistically significant correlations between changes in ADL and gender, education or occupation. For those who were RD or FD at baseline, no significant correlations between changes in ADL were found with gender and occupation. In summary, only among subjects who were FI at baseline, males were more than the females with statistical significance in maintaining independence in ADL. Among the Chinese elderly from 2002 to 2005, subjects who were in the younger age range among the elderly population, who had obtained a higher level of education or who lived in rural areas of China had the highest likelihood of maintaining independence in ADL when comparing with their respective counterparts-the older age range among the elderly population, who had lower education or who lived in urban areas.

On the other hand, among socioeconomic factors, significant correlations were observed between household incomes and change in ADL from all baseline categories. A significant association between occupation and ADL change from baseline were observed only in subjects categorized as FI at baseline. A trend in those with lower socioeconomic status possessing a greater likelihood of maintaining ADL independence was observed. Regarding health factors, both the frequency of serious illness within the past 2 years and the MMSE scores were associated with significant changes in ADL. Among the exposure factors, significant changes in ADL from all baseline categories of ADL were correlated with marital status, living arrangements, the type of caregiver when the subject was ill, or adequate of medical service. In terms of health insurance, having health insurance resulted in maintenance of independence in ADL in subjects who were RI, or RD and FD at baseline. These findings indicate that almost all of the exposure factors influenced change in ADL status according to different ADL levels at baseline.

Table 2 includes three multinomial logistic regression models to test the influence of residence, education, household income, occupation, marital status, living arrangements, and availability of healthcare on changes in ADL from the different baselines for elderly subjects in China, after adjusting for age and gender. The first model estimated the relative risk factors of progressing from FI to $\mathrm{RI}, \mathrm{RD}, \mathrm{FD}$, and dying among the onset of ADL at FI baseline (We indicated them as RI/FI, RD/FI, FD/FI and Dead/FI). The second model estimated the relative factors of progressing from RI to FI, RD or FD, and dying among the onset of ADL at RI baseline (We indicated them as FI/ RI, RD + FD/RI and Dead/RI). The third model estimated the relative factors of progressing from RD or FD to FI or $\mathrm{RI}$ and dying among the onset of ADL at RD or FD baseline (We indicated them as FI + RI/RD + FD and Dead/RD + FD).

Table 2 shows that, after adjusting for age and gender, those who lived in urban areas had a higher risk of ADL decline from FI to RI (OR = 1.46, 95\% CI: $1.22-1.74)$, FI to $\mathrm{RD}(\mathrm{OR}=1.47,95 \% \mathrm{CI}: 1.06-2.04)$ than those who lived in rural areas. Also those who lived in urban areas had a higher risk of not improving ADL from RI to FI $(\mathrm{OR}=0.48,95 \%$ CI: $0.33-0.75)$, and RD or FD to FI or $\mathrm{RI}(\mathrm{OR}=0.49,95 \% \mathrm{CI}: 0.36-0.65)$ than those who lived in rural areas. On the other hand, living in urban areas was found to help protect against dying at $\mathrm{RI}(\mathrm{OR}=0.74,95 \%$ CI: 0.57-0.96) compared with those living in rural areas.

In addition, lower education was found to help protect against a decline in ADL from FI to RI (OR $=0.73,95 \%$ CI: $0.56-0.94)$, RI to $\mathrm{RD}$ or $\mathrm{FD}(\mathrm{OR}=0.42,95 \% \mathrm{CI}$ : $0.25-0.73$; OR $=0.37,95 \%$ CI: $0.20-0.68$ ). Also lower education was found to help improve from RD or FD to RI or FI $(\mathrm{OR}=4.64,95 \%$ CI: $2.21-9.75$; OR $=3.57,95 \%$ CI: 1.56-8.17). However, those who had lower education had a higher risk of dying at RD or FD (OR $=2.34,95 \%$ CI: 1.43-3.81), than those with a higher level of education. 
$\begin{array}{ll}\bar{\delta} & \overline{0} \\ \dot{0} & \dot{v}\end{array}$

$\begin{array}{ll}\bar{\Xi} & \overline{8} \\ \dot{\nabla} & \dot{v}\end{array}$

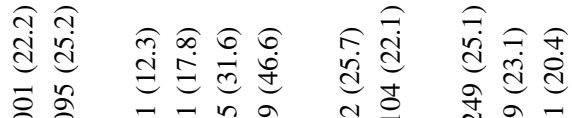

\&

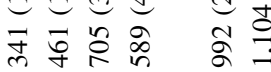

ปे

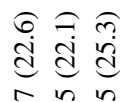

守鱼 $\frac{n}{7}$

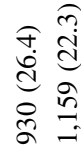

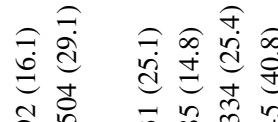

命

ले

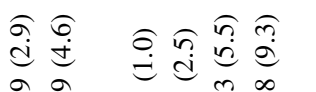

$\begin{array}{ll}\infty & \infty \\ \infty & \infty \\ & \infty \\ y & \infty\end{array}$

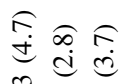

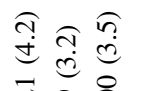

$\begin{array}{ll}\infty & 0 \\ \infty & 0 \\ \infty & \infty\end{array}$

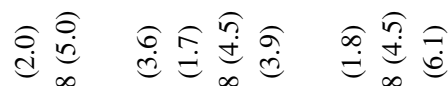

是

그

$\stackrel{\infty}{\infty}$

$\therefore \stackrel{\infty}{\sim}$

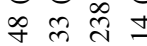

$\forall$ 舟

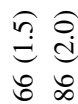

กิ

$\underset{1}{\sigma} \underset{0}{0}$

어욯

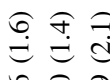

$\underset{\infty}{\oplus} \underset{\sigma}{\sigma}$

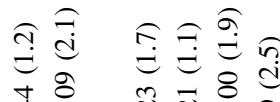

$\widehat{\widehat{T}} \stackrel{\widehat{\vartheta}}{=} \underset{\mathrm{i}}{\mathrm{i}}$

में 의

to $q$ in

और

提

तथ $\bar{\wedge} \circ$

iे

च ते

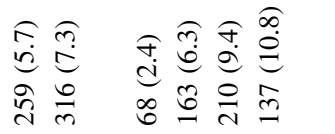

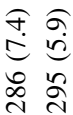

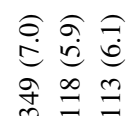

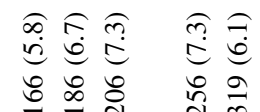

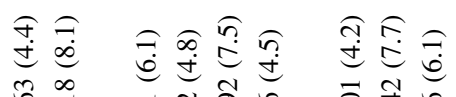

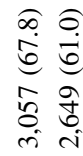

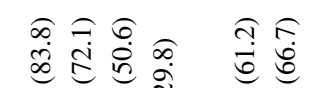

ণิ?

के

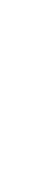

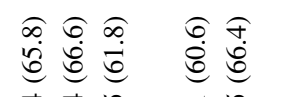

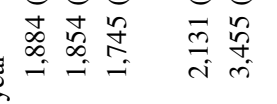

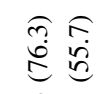

$\begin{array}{cc}m & = \\ \infty & \infty \\ i & i\end{array}$

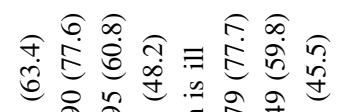
㐫.



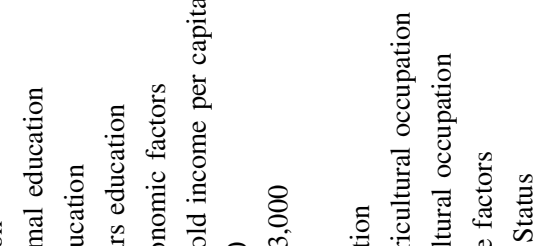

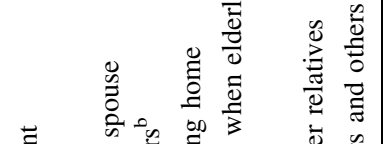

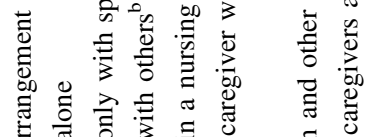

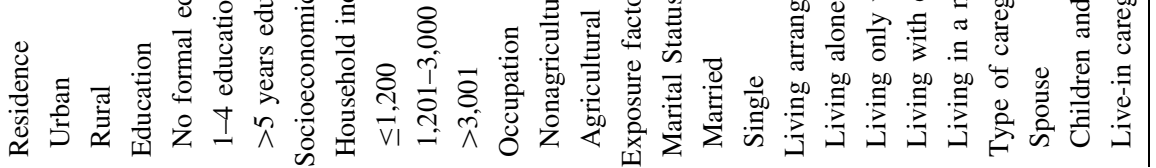




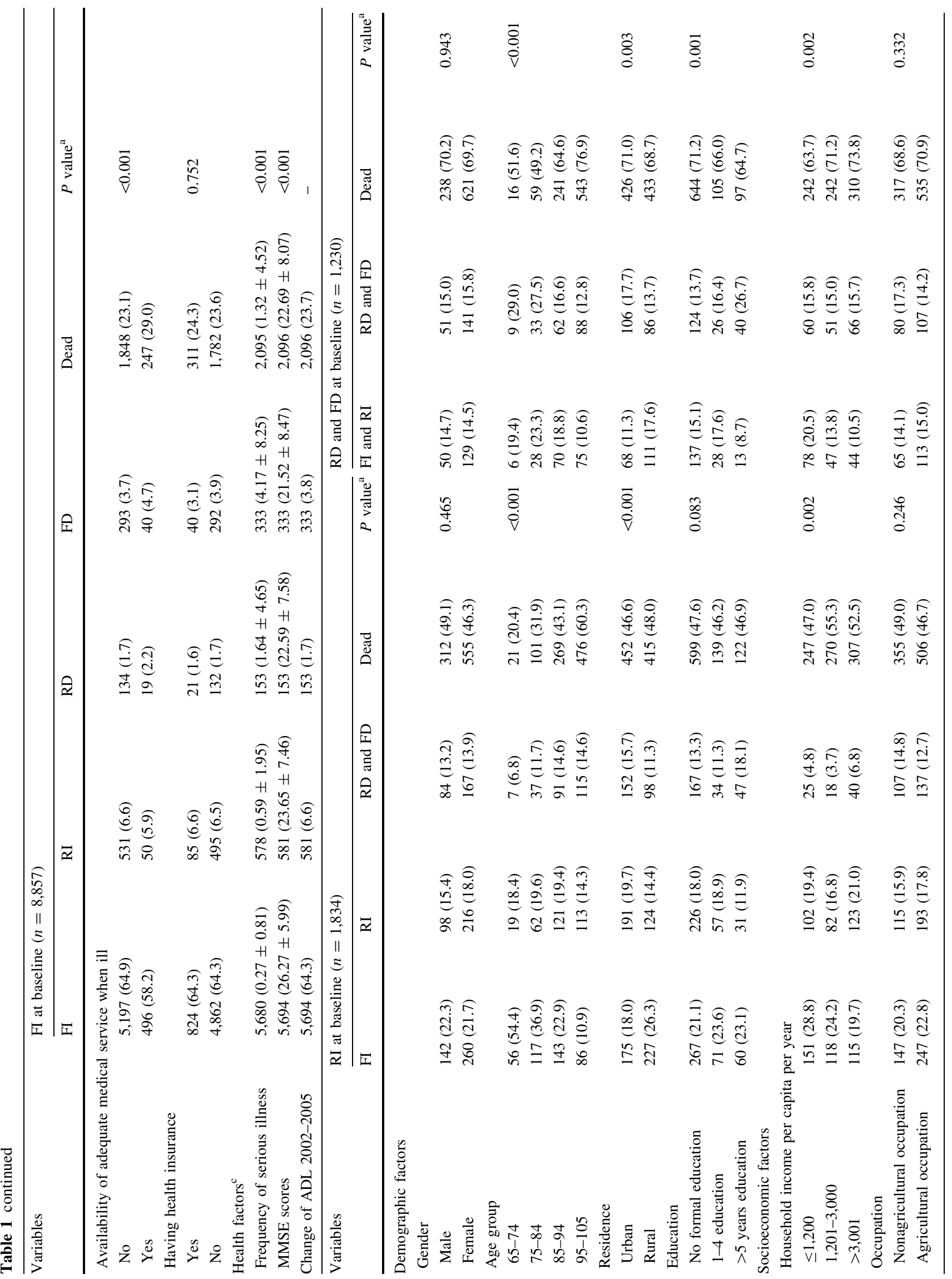




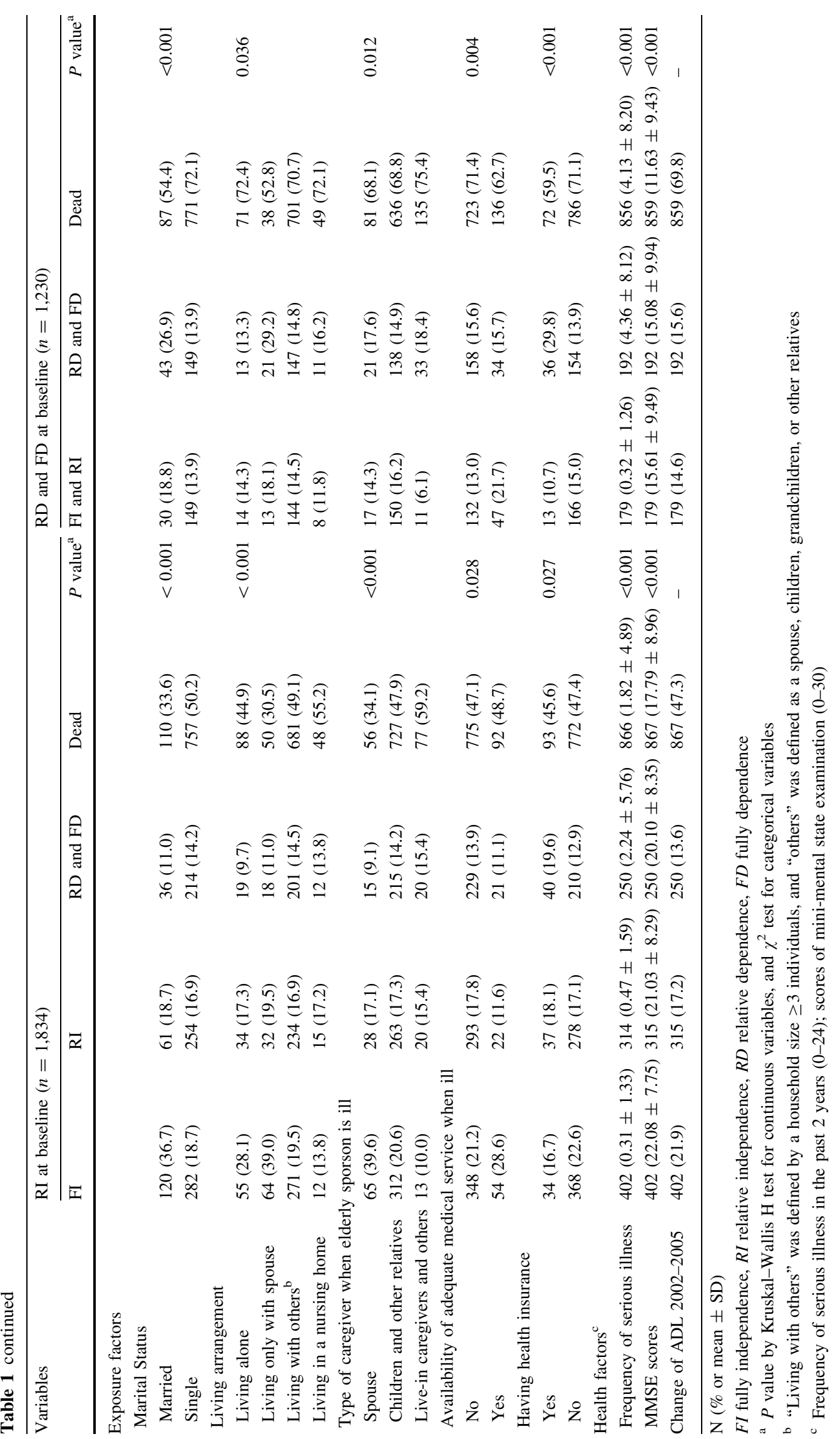




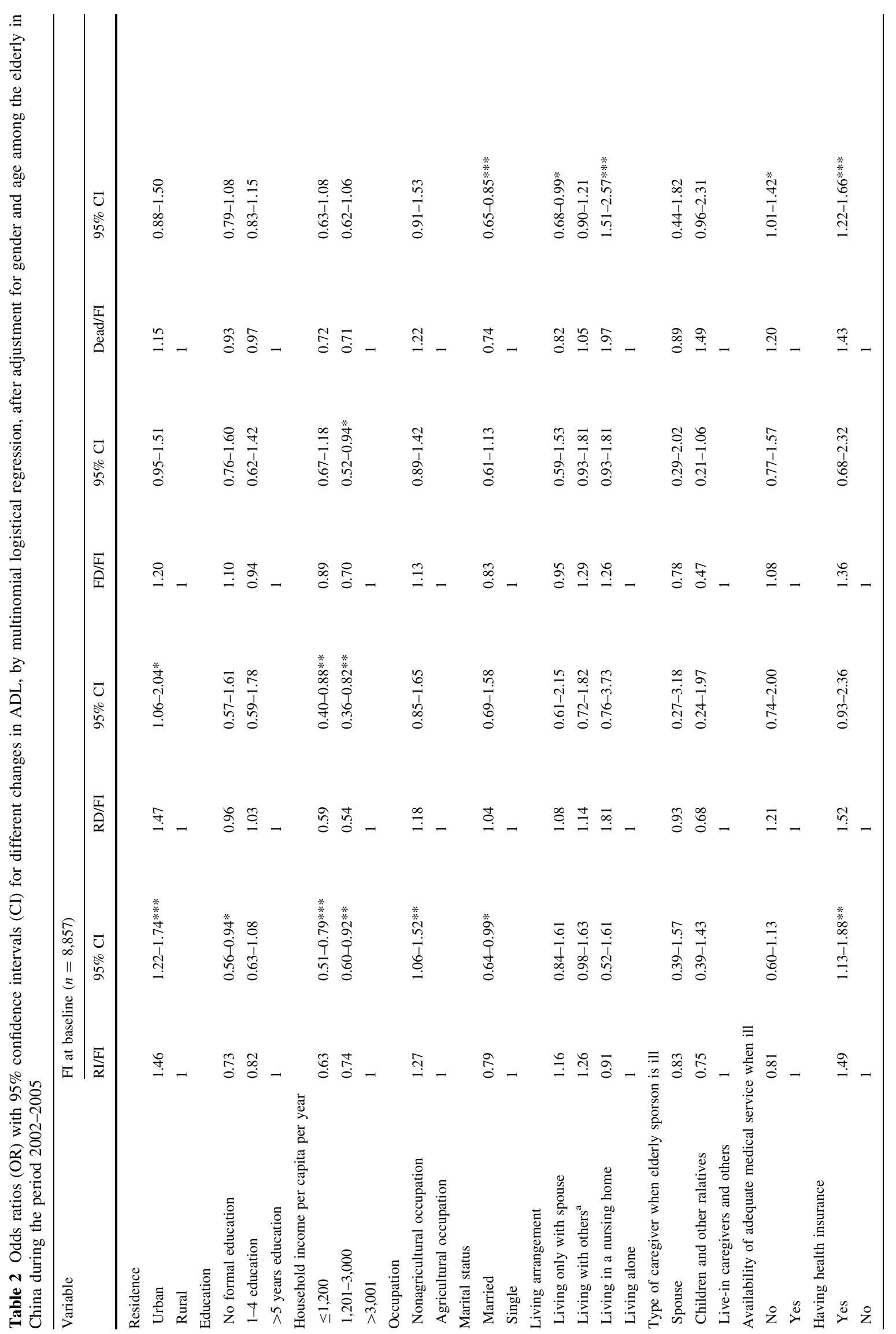




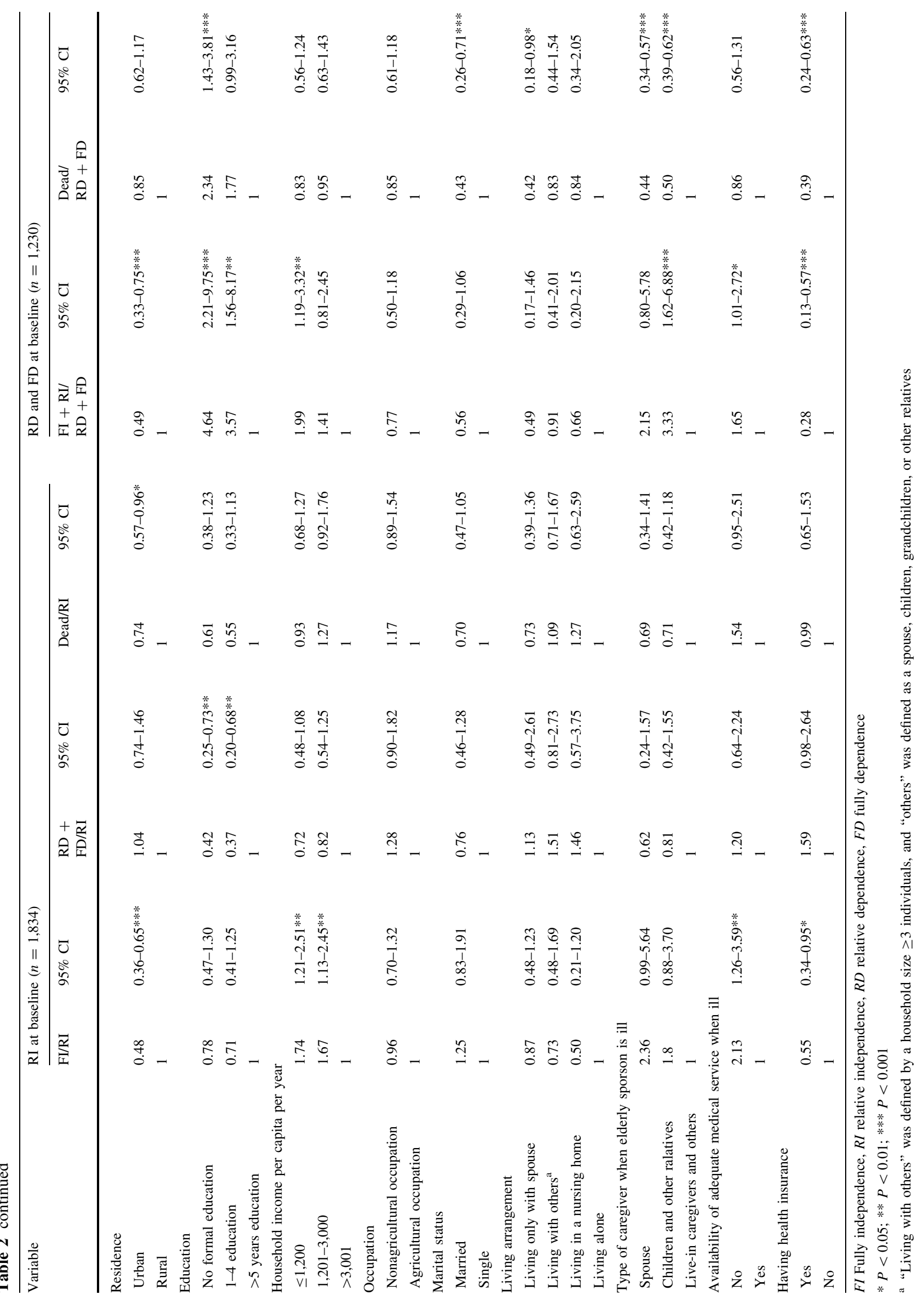




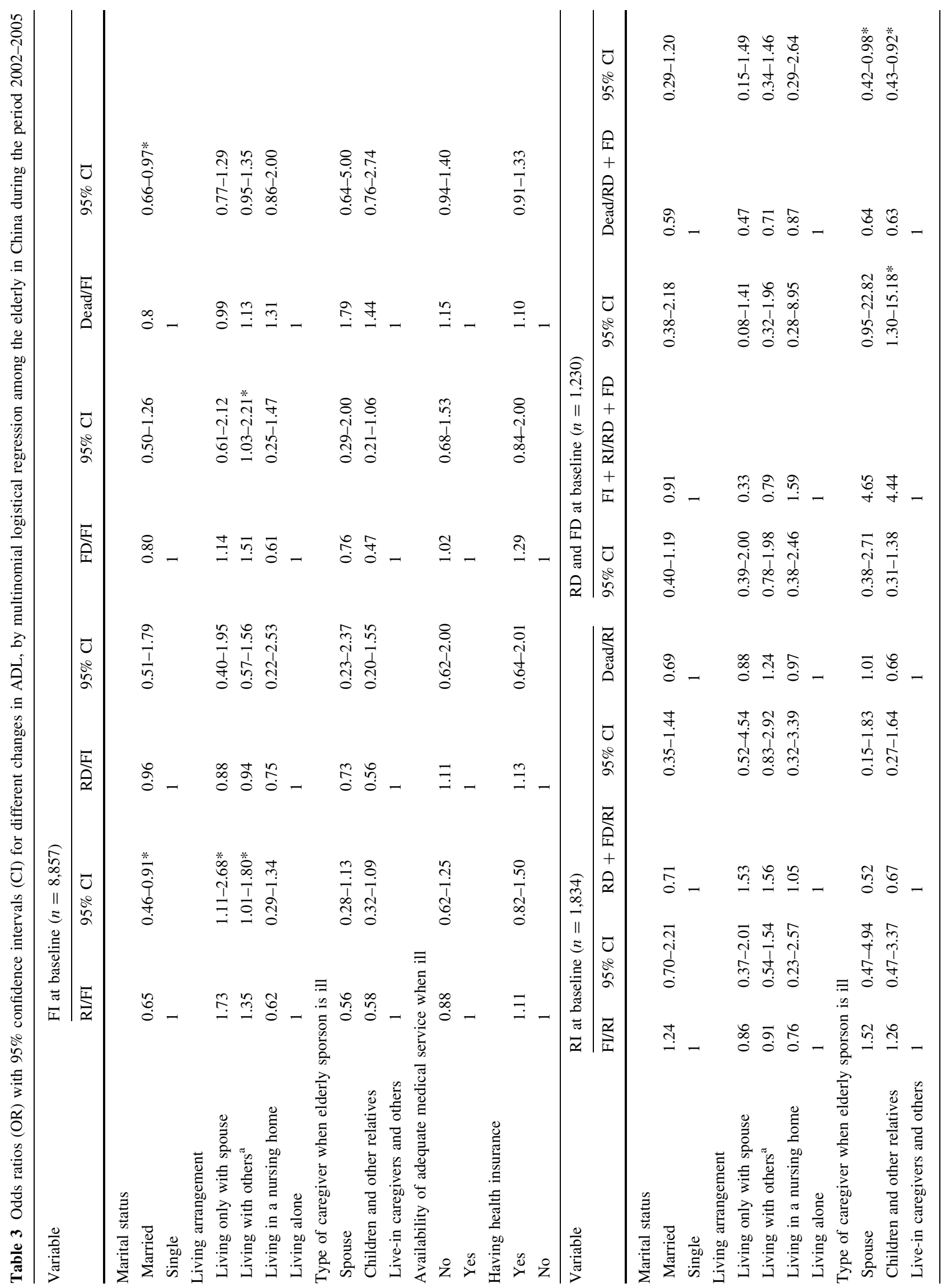




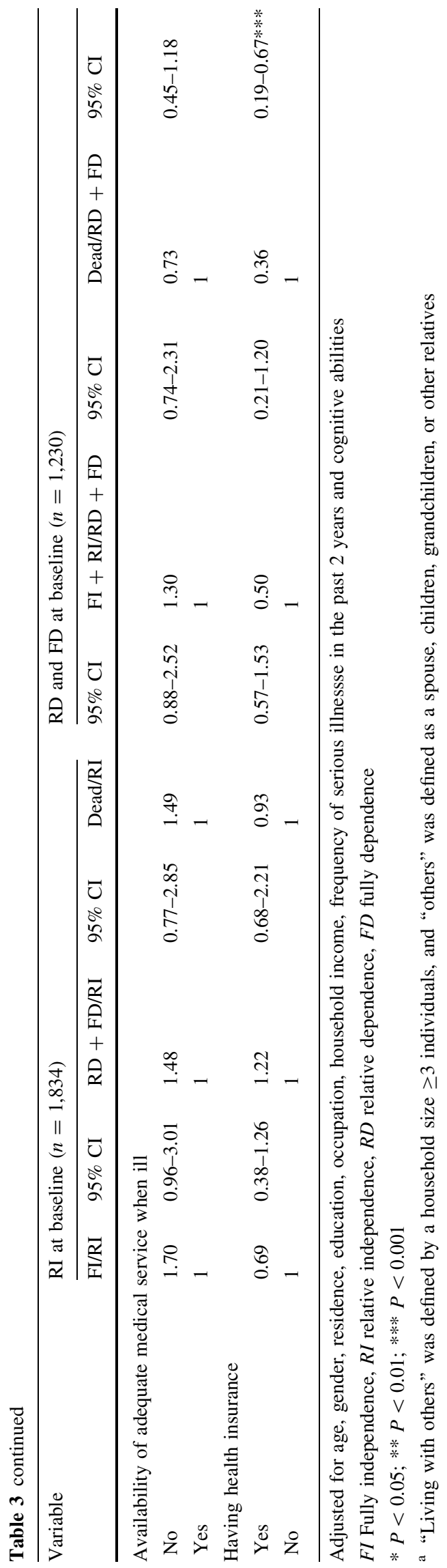

Similarly, lower household income was found to help protect against a decline from FI to RI $(\mathrm{OR}=0.63,95 \%$ CI: $0.51-0.79$; OR $=0.74,95 \% \mathrm{CI}: 0.60-0.92)$, FI to RD $(\mathrm{OR}=0.59,95 \%$ CI: $0.40-0.88 ;$ OR $=0.54,95 \%$ CI: $0.36-0.82)$, FI to FD (OR $=0.70,95 \%$ CI: $0.52-0.94)$, and improve ADL from RI to FI (OR $=1.74,95 \%$ CI: $1.21-$ $2.51 ; \mathrm{OR}=1.67,95 \% \mathrm{CI}: 1.13-2.45)$, RD or FD to FI or $\mathrm{RI}(\mathrm{OR}=1.99,95 \%$ CI: $1.19-3.32)$, compared with those who had higher income. Moreover, those who had nonagriculture jobs before they reached 60 years of age had a higher risk of ADL decline from FI to RI $(\mathrm{OR}=1.27,95 \%$ CI: 1.06-1.52) than those who were farmers before.

Table 2 also shows that, after adjusting for age and gender, being married was found to help protect against a decline in ADL from FI to RI $(\mathrm{OR}=0.79,95 \% \mathrm{CI}$ : 0.640.99). Moreover, those who had health insurance had a higher risk of ADL decline from FI to RI, compared to those who were FI at baseline and lacking health insurance ( $\mathrm{OR}=1.49$, 95\% CI: 1.13-1.88). In addition, among subjects who were FI at baseline, the elderly who were married or living only with spouse had a lower likelihood of death, as opposed to those who were single or living alone $(\mathrm{OR}=0.74,95 \% \mathrm{CI}: 0.65-0.85$; and $\mathrm{OR}=0.82$, 95\% CI: 0.68-0.99, respectively). On the other hand, the elderly who lived in a nursing home, those with health insurance, or those who experienced inadequate medical service when ill were found to have a higher likelihood of death among subjects who were FI at baseline $(\mathrm{OR}=1.97$, 95\% CI: $1.51-2.57$; OR $=1.20,95 \%$ CI: $1.01-1.42$; and $\mathrm{OR}=1.43,95 \%$ CI: $1.22-1.66$, respectively) compared to their counterparts.

For those who were RI at baseline, having inadequate medical services when ill was found to prevent ADL change from RI to FI, compared to those with adequate medical services $(\mathrm{OR}=2.13,95 \%$ CI: $1.26-3.59)$, while having health insurance was found to have a lower rate of ADL change from RI to FI (OR $=0.55,95 \%$ CI: 0.34 0.95). Similarly, after adjusting for age and gender, among those with ADL values of RD and FD at baseline, those who either had children or other relatives as caregivers or experienced inadequate medical service when ill were found to have a higher rate of ADL change from RD or FD to FI or RI, compared to those with unrelated live-in caregivers or who experienced adequate medical service when ill (OR $=3.33,95 \%$ CI: $1.62-6.88$; and $\mathrm{OR}=1.65$, 95\% CI: $1.01-2.72$, respectively). Having health insurance was found to have lower rates of ADL change from RD or FD to FI or RI, compared to those without health insurance $(\mathrm{OR}=0.28,95 \%$ CI: 0.13-0.57). In addition, those who were married or who lived only with their spouse, children or other relatives as caregivers during illness, or those who had health insurance had a less likelihood of death compared to their counterparts $(\mathrm{OR}=0.43,95 \% \mathrm{CI}$ : 
0.26-0.71; OR $=0.42,95 \%$ CI: $0.18-0.98 ;$ OR $=0.44$, 95\% CI: $0.34-0.57$; OR $=0.50,95 \%$ CI: $0.39-0.62$; and $\mathrm{OR}=0.39,95 \%$ CI: $0.24-0.63$, respectively).

Multivariate-adjusted ORs for marital status, living arrangements, and healthcare issues affected changes in ADL, according to the baseline category of ADL, as is shown in Table 3. After adjusting for main confounding variables, married elderly who were FI at baseline were less susceptible to experiencing ADL change from FI to RI status or death $(\mathrm{OR}=0.65,95 \% \mathrm{CI}$ : $0.46-0.91$; and $\mathrm{OR}=0.80,95 \%$ CI: $0.66-0.97$, respectively) than those who were single. In addition, elderly who lived with just a spouse or who lived with children and/or others (sometimes including a spouse) had a 1.73- and 1.35-fold higher risk, respectively, towards ADL change from FI to RI status, and a 1.51-fold higher risk of ADL change from FI to FD status, compared to those who lived alone. Notably, once the elderly showed dependency in ADL function, living alone was no longer a significant predictor of future ADL status. For those who were RD or FD at baseline, after adjusting for main confounding variables, elderly who were cared for by children and other relatives during illness were 4.4 times more likely to experience a change in ADL from RD or FD to RI or FI, compared to those with unrelated live-in caregivers. In addition, among those who were RD or FD at baseline, the elderly who were cared for by a spouse, or by children and other relatives during illness, or who had health insurance had a lower likelihood of death $(\mathrm{OR}=0.64,95 \%$ CI: $0.42-0.98$; OR $=0.63,95 \%$ CI: $0.43-0.92$; and $\mathrm{OR}=0.36$, 95\% CI: $0.19-0.67$, respectively). Other factors did not significantly affect the change in ADL.

\section{Discussion}

Given the paucity of previous research conducted on the impact of marital status, living arrangements, and healthcare availability on change in ADL among Chinese elderly, the current study may have important implications for policy-making regarding community and family support systems. Specifically, we found that Chinese elderly who are married are less likely to experience ADL decline and have a lower risk of death, compared to their single counterparts. Among those who were FI at baseline, the Chinese elderly who lived alone were less likely to experience ADL decline compared to those who lived with their spouse, or lived with children and/or other people (including a spouse). Another study of Chinese people $\geq 55$ years of age showed similar results; the elderly who lived with people other than a spouse had a significantly higher risk of decline in functional status than did elderly who lived alone or with only their spouse [12].
The following reasons might explain these findings. First, a recent survey conducted in China found that more elderly people who were financially and physically independent preferred to live either alone or with their spouse. This trend was especially true for urban elderly with high levels of education and who had held white-collar jobs before they reached 60 years of age [2, 13]. Most of these subjects enjoyed a full life. Thus, continuing to live alone or with just their spouse seemed to be beneficial in maintaining ADL, compared to other elderly people who lived with children or others.

Secondly, public pensions in China currently are reserved for only a small proportion of the elderly population [14-16]. Therefore, in both urban and rural areas, many elderly people provide their adult offspring with housing, housework, or care of grandchildren in return for financial and other material support. The degree to which an adult offspring would provide financial support to their elderly parents is dependent on the parents' need [14]. Such family support compensates for insufficient access to public resources. For elderly who live with others, including offspring, the long-term intergenerational exchange may result in physical fatigue, leading to a decline in physical functioning.

Third, according to our data, the average age of married elderly in China is lower than that of single elderly, and the majority of married elderly in China are male, while the majority of those who live alone are female. From our data, we surmise that married male elderly in China were less likely to experience deteriorating function in ADL and had a lower likelihood of death compared to those who are single. On the other hand, elderly women living alone in China were less likely to experience ADL decline. Similar studies in Japan and western countries [17, 18] indicated that males who lived alone had higher health risks, while women who lived alone had significantly lower mortality rates. In sum, further study is required to examine the benefits of marriage with regard to maintenance of ADL independence by gender and age in China.

Finally, living alone allows elderly people to learn to maintain a high level of independence, a phenomenon referred to as a "biologically conditioned reflex" [19]. According to our analyses, compared to those who live with others, elderly people who live alone are more independent in not only the six basic ADL activities (eating, dressing, indoor mobility, bathing, using the toilet, and continence), but also in instrumental ADL activities, such as walking outside, shopping, preparing meals, or cleaning.

Our study revealed that once an elderly person becomes functionally dependent on others, the status of living alone is not a significant predictor of further functional decline. After adjusting for confounding factors in those who are RD or FD at baseline, the elderly who were cared for by 
children and other relatives during illness were 4.4 times more likely to experience a change in ADL from RD or FD to RI or FI, compared to those with unrelated live-in caregivers. In addition, the elderly who were cared for by a spouse, or children and other relatives had a lower likelihood of death, compared to those with unrelated live-in caregivers. These findings imply that currently, the majority of elderly in China still rely on traditional forms of family support. Compared to the past, a greater proportion of modern-day elderly people in China are single or live with just their spouse [2], but once the elderly become functionally dependent, they usually move to live with their adult offspring and other people [20, 21]. According to traditional confucian-based family values in China, the happiest elderly are those surrounded by as many descendants as possible, especially during the last stages of their lives. Spouses, children, and other relatives who play the role of caregivers when an elderly relative becomes ill is considered to be common [13]. Moreover, compared to having an unrelated, live-in caregiver, having spouses, children, and other relatives who play the role of caregivers will better protect the elderly from psychological isolation and will provide sick elderly people with adequate medical services. However, according to the studies, even after the introduction of long-term health care insurance in 2000 in Japan, the potential supply of offspring caregivers present varied substantially by gender, marital status, and opportunity costs of children [22]. Judging by this result found in Japanese studies, this study also implies that professional training for live-in caregivers is required due to the rapidly increasing proportion of the aged within the population in China, partially as a consequence of the one-child policy.

Our study also indicated that among those who were $\mathrm{RD}$ or FD at baseline, Chinese elderly with health insurance had a lower likelihood of death. Unfortunately, in recent decades, the elderly in China have experienced inadequate medical service or treatment due to reforms in the medical care system. From 1949 (establishment of new China) until the end of the first decade of the twenty first century, the healthcare system in China has experienced the elimination of private medical facilities, the rural cooperative medical system (i.e., the presence of "barefoot" doctors), and the public and private healthcare systems. Since the economic reform that occurred in the 1980s, public and private healthcare systems have emerged once again. However, the quality of healthcare practice has deteriorated due to poor management and less government regulation of the medical market [23, 24]. Most elderly people in China have experienced inequality in healthcare, because of their low socioeconomic status and the low quality and availability of health insurance. Based on our data, only 1,606 (14\%) elderly people in this study had health insurance and most of them live in urban areas of China; about one-third had a household income of less than 1,200 RMB per capita per year, and most of them live in rural areas of China.

However, in this study, residing in rural areas, and having lower education or lower household income appear to be a protective factor against dependency on others for assistance with ADL. On the other hand, residing in urban areas or having higher education helped protect against progressing ADL from RI, RD or FD to dying compared with their counterparts (see Tables 1, 2). In addition, elderly who experienced inadequate medical service when ill or lacking health insurance were found to have a lower likelihood of ADL decline (see Table 2). This could be explained by the "select bias" [10], selecting for those with a lower socioeconomic status, lower availability of health insurance, more difficult life experiences, and higher mortality at younger ages in rural regions of China. As a result, the selected elderly in rural areas might be with stronger survival skills than those in urban areas in China.

In addition, due to a massive shift from rural to urban areas among the younger population, a large proportion of the elderly population remains with their grandchildren in rural villages $[25,26]$. Number of adult offspring willing to take on care-giving responsibilities for aging parents is declining in modern China. As a result, the elderly in China are once again facing disadvantages in healthcare and assistance. All of these factors suggest that health insurance for the elderly should be established and that adequate medical service or treatment for the Chinese elderly should be provided as soon as possible.

The present study has several methodological limitations. Some factors of measurement were not sensitive enough to detect a functionally independent status of the elderly. For example, those considered to be married included those currently married and living together as well as those currently married and living separately. It would be better to distinguish the difference between these two groups. However, there were only 295 (6.3\% of all married subjects) elderly subjects who were currently married and living separated. In addition, the living arrangements in the present study were only categorized as living in a nursing home, living alone, living with just a spouse, and living with "others" ("others" was defined as a spouse, children, grandchildren, or other relatives). Future studies should include more detailed classifications of marital status and living arrangements. Future studies should also assess how changes from different baselines of ADL can be explained by the differences between those who experienced physical decline and changed their living situation from a small family to a large family, and those who experienced a decline in functional status, but did not change their family structure within their household. Moreover, short period of observation (2002-2005) may be one of methodological 
limitation. As a result, reverse causality might occur in some items.

Interpretation of our findings should consider all of these limitations. Our study is still unique, however, due to our application of nationwide data that clarified the impact of marital status, living arrangements, and healthcare issues on changes in ADL among the elderly in China. We conclude that within the surveyed time period, the majority of elderly people in China still relied on a traditional type of family support. As the elderly have different functional levels, healthcare policies in China should consider both community and family support systems.

Acknowledgments Collection of the CLHLS datasets used in the current study was supported by the NIA/NIH, China Natural and Social Sciences Foundations, the UNFPA and the RGU. We thank Professors Zeng Yi, Gu Danan (Duke University Center for Study of Aging and Human Development/Medical School and Peking University Center for Healthy Aging and Family Studies/China Center for Economic Research), and Liu Yuzhi (Center for Healthy Aging and Family Studies and Institute of Population Research, Peking University).

\section{References}

1. Zeng Y, George L. Extremely rapid aging and the living arrangement of elderly persons: the case of China. In: Living arrangements of older persons, Population Bulletin of the United Nations, Special Issue. New York: United Nations; 2002. pp. $42-3$.

2. Hu L. Transition from tradition to modern-the analysis of the characteristics and the causes about Chinese family structure. Northwest Popul. 2004;2:29-31. (in Chinese).

3. Rowland DT. Family characteristics of internal migration in China. Asia Pac Popul J. 1992;7:3-26.

4. Zyzanski SJ, Medalie JH, Ford AB, Grava-Gubins I. Living arrangements and well-being of the elderly. Family Med. 1989;21:199-205.

5. Joung IM, van de Mheen H, Stronks K, van Poppel FW, Mackenbach JP. Differences in self-reported morbidity by marital status and by living arrangement. Int J Epidemiol. 1994;23:91-7.

6. Sarwari A, Fredman L, Langenberg P. Prospective study on the relation between living arrangement and change in functional health status of elderly women. Am J Epidemiol. 1998;147:370-8.

7. Fung HH, Stoeber FS, Yeung DY, Lang FR. Cultural specificity of socioemotional selectivity: age differences in social network composition among Germans and Hong Kong Chinese. J Gerontol B Psychol Sci Soc Sci. 2008;63:156-64.

8. Chinese Longitudinal Healthy Longevity Survey (CLHLS). http://www.geri.duke.edu/china_study.
9. Research group of healthy longevity in China. Data collections of the healthy longevity survey in China 1998. Beijing: Peking University Press; 2000.

10. Zeng Y, James WV, Xiao ZY, Zhang CY, Liu YZ. The healthy longevity survey and the active life expectancy of the oldest old in China. Popul Engl Selection. 2001;13:95-116.

11. Katz S. Studies of illness in the aged. The index of ADL: a standardized measure of biological and psychological function. J Am Med Assoc. 1963;185:94ff.

12. Beydoun M, Popkin M. The impact of socioeconomic factors on functional status decline among community-dwelling older adults in China. Soc Sci Med. 2005;9:2045-57.

13. Li WL. Aging and welfare policies in China. Sociol Focus. 1998;1:31-43.

14. Lee YJ, Xiao ZY. Children's support for elderly parents in urban and rural China: results from a national survey. J Cross Cult Gerontol. 1998;13:39-62.

15. Nee V. Social inequalities in reforming state socialism: between redistribution and markets in China. Am Sociol Rev. 1991; $56: 267-82$.

16. Walder AG. Property rights and stratification in socialist redistributive economic. Am Sociol Rev. 1992;57:524-39.

17. Murata C, Takaaki K, Hori Y, Miyao D, Tamakoshi K, Yatsuya $\mathrm{H}$, et al. Effects of social relationships on mortality among the elderly in a Japanese rural area: an 88-month follow-up study. J Epidemiol. 2005;15:78-84.

18. Gliksman MD, Lazarus R, Wilson A, Leeder SR. Social support, marital status and living arrangement correlate of cardiovascular disease risk factors in the elderly. Soc Sci Med. 1995;40:811-4.

19. Schmajuk N, Larrauri J. Associative models can describe both causal learning and conditioning. Behav Processes. 2008; $7: 443-5$.

20. Zhao YX, Wang DW. Investigation on attitude and influence factors of elders' non-familial support. Chin J Public Health. 2007;23:261-3. (in Chinese).

21. Sugisawa H, Nakatani Y, Shimizu Y. A study of family support, health state and medical care among functionally dependent elderly in Shanghai, China. Nippon Koshu Eisei Zasshi. 1991;38:546-52. (in Japanese).

22. Hanaoka C, Norton EC. Informal and formal care for elderly persons: how adult children's characteristics affect the use of formal care in Japan. Soc Sci Med. 2008;23:65-74.

23. Liu G, Liu XZ, Meng QY. Privatization of the medical market in socialist China: a historical approach. Health Policy. 1994; 27:157-74.

24. Liu Y, Rao K, Hsiao WC. Medical expenditure and rural impoverishment in China. J Health Popul Nutr. 2003;21:216-22.

25. Li SM, Siu YM. A comparative study of permanent and temporary migration in China: the case of Dongguan and Meizhou, Guangdong Province. Int J Popul Geogr. 1997;3:63-82.

26. Giles J, Mu R. Elderly parent health and the migration decisions of adult children: evidence from rural China. Demography. 2007;44:265-88. 